\title{
Bienes comunes y diseño temitonial autónomo
}

\author{
Femando de la Cuadra Arancibia \\ Universidad Católica del Maule, Talca, Chile. \\ Email: fdelacuadra@ucm.cl \\ Beatriz Cid Aguayo ${ }^{1}$ \\ Universidad de Concepción, Concepción, Chile. \\ Email: beatrizcid@udec.cl \\ Eduardo Letelier Araya \\ Universidad Católica del Maule, Talca, Chile. \\ Email: eletelier@ucm.cl
}

El presente número esuna colección de escritosy casos sobre procesos de comunalización económica existentes en nuestros teritorios. En su conjunto dan cuenta del reconocimiento empíric o -y también del deseo- que no todas las esferas de la vida económic a están completa mente subsumidas a las rela cionesy lógic as de la s rela ciones de ca pital. Esta reflexión se entronca en una amplia tradición heterodoxa en el pensamiento que algunos llaman «susta ntivista», otros «anti-utilita rista» y más recientemente «performa tiva » que han problematizado el objeto de «a economía»como ciencia y el «mercado autorregulado» como práctica, en tanto «inventan» un discurso naturalizado a la vez que un horizonte nomativo porel cual se organiza la realidad (Polanyi, 1957). La ciencia económica neoclásica se ha construido sobre la base de un modelo de acción y decisión, que no da cuenta de la diversidad de subjetividades -intereses a pasionados que a niman lo económico. Más aún, la economía-disciplina ha construido la economía-cosa (La tour y Lépinay, 2008), en lo que Polanyi (1957) denominó una gran profecía auto-cumplida. La economía-práctica se entiende a sí c omo el resulta do de las dec isionesy a c cionesque cursa mosy donde el rango de racionalidades es más a mplio y diverso que la abstracción conceptual modema y fuertemente masculinista del hommo económicus.

Se releva así lo performativo de la actividad económica -como proceso que se está haciendo, lo que deja abierta distintas posibilidades de futuro-y la relevancia de los actores frente a estruc turas natura liza das. Con ello devuelve la capacidad de acción e incidencia política de los actores y contribuye a un discurso económico empoderador. Este cambio semántico, rechaza la naturalización de los discursos de la dominación, poder y violencia para reconstruir un disc urso capaz de vislumbrarguiones a ltema tivos. Así el foco de a nálisis deviene en problema tizarcolec tiva y partic ipativa mente práctic aseconómic a sétic amente a rraiga das ya existentes en una política de comunalización y construcción de diseños temi-

\footnotetext{
${ }^{1}$ Los editores invitados, Beatriz Cid y Eduardo Letelier se encuentran ejecutando en este momento el Proyecto Fondecyt $N^{\circ} 1190020$ Comunalización y hetereogenidades económicas: espacios de diálogo en tomo a casos en el centro-sur de Chile.
} 
toriales-como los que se describen en este volumen- que permita modelarlas y a mplific arlas hacia otros espacios temitoriales.

Esta discusión a su vez foma parte de una discusión ética amplia en tomo a cómo el mercado autorregulado ha sacado la esfera de la producción, del flujo de la vida, de la materia y la energía. Diversos proyec tos teóric osy polític os han problema tiza do este discurso natura lizado: las economías socia les y solidarias, las propuestas de decrecimiento, de otro desa rollo, de altemativas al desa rollo en Améric a del Sur, la economía ecológica, así como también el trabajo sobre heterogeneidad económica. En su conjunto estas visiones relevan la diversidad de formas económicas existentes en contextos geográfic os e históricosvaria dos. Son perspectivas crític asque, frente a la expansión del proceso de privatiza ción genera lizada de la vida, proponen la creación, defensa y reinvención de loscomunescomo camino para evitar la «tragedia de lo no-común» (Laval y Dardot 2014). Se busca asíampliar las posibilida des de reconocer otras relaciones e ima gina r otras formas de existir.

La pregunta por los comunes es antigua en la economía - disciplina. Desde la teoría neoc lásica, los bienes comunes son definidos como bienes de libre acceso (no excluibles) pero que son agotables (rivales en el consumo). Se diferencian de los bienes privados (excluibles y riva les en el consumo), de los bienes públicos (no excluibles y no rivales en el consumo) y de los bienesde club o colectivos (excluiblesy no rivalesen el consumo). Mucho de la literatura económic a tradicional los presenta como bienes en peligro de agotamiento -en riesgo pemanente de tragedia-por el mal uso que usuarios ambiciosos, egoístas y desarticulados: bien de todos, es bien de nadie, y va a tender a desaparecer. Desde esta perspec tiva solo pueden sercuidadosa tra vés del ejercic io de una regula ción esta tal superior o bien a tra vés de asignación de derechos claros de propiedad. Vemosacá una doble tragedia: dejar a los comunes en manos de Estados cuya eficiencia teritorial es dudosa, o bien en manos de priva dos que pueden no estar interesa dos en su cuidado sino solo en su rendimiento. El trabajo de recopilación desarrollado por Elinor Ostrom (1990) reconoce sin embargo una salida a este dualismo, en tanto observa que arreglos instituc ionales colectivos logran asegurar su mantención y previenen la provisión privada sub-óptima de los mismos, evitan el problema del polizonte (free riding), volviendo innecesaria la intervención estatal (Ostrom, 1990).

Desde una perspectiva sustantiva, a nti-utilita ria y performativa los bienescomunes pueden ser definidos como aquellos en cuya creación, uso, consumo, gobemanza, circulación y ampliación participa más de un individuo. Esta visión convoca a entenderlos no como un conjunto delimitado de bienes naturales, sino como resultado de prácticas polític as colectivas y genera tivas que los crean, cuidan y expanden permanentemente. La existencia de bienes comunes - desde bosques y pesca hasta los productos digitales de la economía inmaterial - no son extemos a las personas, sino que están entrela za dos a los sujetos comunita rios que los construyen y gestionan frente a los sucesivos cercamientos priva tizadores. Existe así una dialéctica entre procesos de privatiza ción y comuna lización: muchos bienes comunes se crean como tales ante la amenaza de la privatización o a gotabilidad. 
stos procesos son de alta relevancia polític a pues reivindican los comunes y la comunidad contra las nuevas formas de apropiación privada y estatal; articulando luchas y prácticas en tomo a lo material y lo inmaterial. Así, frente a la «tragedia de loscomunes», se subraya la puesta en práctic a de lo común, apelando a una comunidad de agentesque producen, mantienen y hacen circula rcomunes en un proceso de «comunalización». De esta manera si la comunaliza ción se refiere a la produc ción y reproduc ción permanente de los comunes, la comunidad esentoncesel resulta do de un proceso de asocia ción y conciencia temitoria l. Esto es $\ll E \mathbb{E l}$ 'nosotros' que produce y comparte estos bienes comunes no es simplemente la a sociación de humanos sino un colectivo de seres humanos y no humanos, incluyendo los ńos y los cuerpos de a gua, las plantas y las selvas, lospec es y lasespeciesa nimales- a lasque nosreferimosfrecuentemente como recursosnaturales [...]»(G ibson-Graham et al. 2017, p. 176).

A partir de estas definiciones una economía comunaliza dora es lo que hacen los a gentes construyendo, gobemando y circulando comunes, así como las relaciones subyacentes a estas agencias. Esto implic a negociaciones éticas y toma de decisiones políticas sobre lo que tradicionalmente se ha denominado problema económico (cómo cuidar los bienes comunes, que producir, como consumir para vivir bien colec tivamente, cua nto exc edente producir y cómo repartirlo). En ella, se reconoce y negocia nuestra interdependencia con otros seres humanos, otras especies y el entomo biofísico de manera autónoma. Es en el proceso de negociación y agencia colectiva para el mantenimiento, creación y circulación de comunes donde se construye comunidad, una srela ciones comunita ria s soc io-na turales, una red de interdependencia entre agentes (humanosy no-humanos) en un determinado territorio.

En resumen, la convocatoria del presente número de la Revista Polis se propuso recibir a portes de artíc ulosque dieran cuenta del rec onocimiento de ejerc ic ios de comunalización en tomo a ciertas a ctivida des ec onómic as espec íficas -ta les como la gestión colectiva de bienes c ultura les y na tura les a so c ia dos al patrimo nio vitiviníc ola, la textileńa, la s semillas y el agua, el atractivo tuństic o entre otros-, que sin embargo son marginadas, desconocidas y exotizadas por el modelo de desarrollo hegemónico.

En partic ular, se busc a ba comprendery problema tiza restos ejerc ic ioso experiencia sdesde la perspectiva de sus limitaciones y potencialidades pa ra proyectarse más allá del espa cio local y constituir la posibilidad de diseños temitoriales autónomos. Para esto se procuró indagaren el modo como estosejerc ic iosela boran a rreglos-sa beres, experiencias y tec nologías- que hibridizan en forma más o menos fluida, saberes y prácticas tra dicionales con tec nologías y conoc imientos modemos, los cuales son potenc ia Imente valiosos, replic ables yescalables.

Estos han sido precisamente los temas principales abordados por el conjunto de los estudios que son parte del presente número monográfic o de la revista. En el primer artículo de esta sección, La recuperación de los comunes en el sur-austral: construcción institucional de Espacios Costeros Marinos de Pueblos Originarios (ECMPOs), los a c a démic os Florencia 
Diestre de la Barra y Francisc o Ara os Leiva, de la Universidad de Chile y de LosLagosrespectivamente, muestran cómo la degradación de los comunes en el sur austral de Chile ha sido propicia da por el sistema de concesiones acuíc olas para la sa Imonic ultura.

En este contexto, desde un enfoque de ecología polític a neo-institucionalista, el a ríc ulo discute el proceso de construcción institucional para la recuperación de los comunes impulsa do en el marco de la implementación de la Ley 20.249, que entrega la administra ción de la zona manino costera a comunidades o asociaciones mapuche lafkenche de la zona sur a ustral de Chile. A través del estudio de tres casos que comparten un Área Geocultural Costera Marítima, los a utores muestran cómo son defendidos los recursos comunes de formas productivas perjudiciales tanto para la zona marino costera como para el habitarde las comunidades.

En partic ular, al considerar la interrela c ión histórica de fac toresa mbienta les, so cia les, físic os demográficos y tecnológicos que inciden en el precio relativo de los recursos naturales a socia dosal mar, el a rtíc ulo hace visibles la sa c tua les a simetrías de podery la gesta ción de nuevascondiciones de poder, graciasa la consolidación de losECMPOs. Del mismo modo, al a na lizar la relación entre ideologías y selección de instituciones, a través de discursos y narrativas de los grupos de interés, el artíc ulo permite comprender los argumentos que legitiman el comportamiento de los actores sobre el control y uso de loscomunes, relevando la forma en que lo comprenden y conceptua lizan sus demandas y establecen compromisos y a lianzas en los proc esos de negociación, a fin de a lc a nzar sus intereses en la gestión de dic hoscomunes.

En el segundo trabajo de esta sec ción, el investigador Martín Lanca man Cárdenasaborda el tema de la Pluralidad de significado en los bienes comunes: mirada Mapuche hacia la defensa de las aguas. Se trabaja a partir del conflic to soc iocultural a socia do la proyec ción y construcción de centrales hidroeléctricas en temitorio mapuche. Las comunidades involucradas han artic ulado la defensa política de sus sistemas de aguas movilizando el termino ngen (entidad espintual dueña del espacio), concepto que da cuenta de la experiencia mapuche del teritorio.

La comprensión de estos otros lenguajes y sus raciona lida des, pemite rec onocer otros universos de sentido -en la concepción de pluriversos trabajada por A. Escobar y M. de la Cadena- y como tal permite trascender un multiculturalismo neoliberal extractivo y neocolonial, para favorecer una construcción multic ultural negocia da sensible a la exigencia de justic ia histórica. Esta s reflexiones problema tizan ta mb ién las visiones c lásicas de bien común para darlugara la mirada de pueblos indígenas en este caso -el pueblo mapuchede aproximación a los teritorios y su política.

El tercer artículo de la serie es de los investigadores mexicanos Mauricio López Ba reto y Miguel Pinkus Rendón de la Universidad Autónoma de México, sede Mérida. En su cola boración titulada Indicadores bioculturales en proyectos de gestión ambiental. E caso de la 
meliponic ultura en Yucatán, ellos desa rrolla n un a nálisis etnog ráfico de un proyec to pa ra el rescate de la meliponic ultura (abeja nativa) en la península de Yucatán, y a partir de ahí con el a poyo de las teorías de valora ción relacional- se proponen indicadores para conocer la incidencia en la dimensión biocultural en proyectos que afectan directamente las prácticas de los comunes socio-ambientales.

El tipo de instrumentos propuestos, se a rraigan en el lugar y el contexto particular de los proyectosconsiderados, siendo de esta manera emergentes y contingentes a l conocimiento y experiencia cultural local. Estos indicadores se alejan expresamente de la valoración moneta ria, con el propósito de a poyar la desmerca ntiliza c ión de los comunes biocultura les, que son amplio, complejos y heterogéneos.

En la cuarta contribución, Christiane Marques Severo y Alessandra Matte de la Universidad Federal de Rio de J aneiro y de la Universidad Tec nológica Federal de Para ná, Bra sil, respectivamente, abordan la temátic a que lleva portítulo Comunalização da gestão do sistema socioecológico do bioma Pampa: análise de um coletivo de pecuaristas tradicionais do Unuguai. El estudio hace una precisa caracteriza ción de las actividades realiza das por gana deros a so ciados con el a poyo de las instituciones pertinentes, lo cual les permite garantizar un tipo de producción sustentable que asegura la conservación y la capacidad de reproducción na tural de los recursosutiliza dos, en este caso, de los pastospara la actividad pecuaria.

En ese sentido, la investiga ción rea liza da se inserta en el debate en tomo a los procesos de gestión de los bienes comunes a partir del a ná lisis del comporta miento de a c to res pertenecientes a las comunidades de ganaderos a sentados en el socioecosistema del llamado Bioma Pampa. Este grupo ganaderos pueden ser considerados como formando parte en un Sistema Socioecológico en la medida en que mantienen una relación sustentable con los rec ursos na tura les utiliza dos en su a c tividad, evitand o el c olapso de dichos ec osistemas, contando también para ello con el apoyo de especialistas, académicos y agentes instituciona les del Esta do urugua yo. En este estudio y en otros a nteriores, las a utoras destacan la presencia de mecanismos de coordinación interinstitucional y de canales efectivos de participación social que tienen un papel signific a tivo en la sustentabilidad del uso de los recursos, lo cual, junto con la estabilidad política e institucional, se refleja en el proceso de planific a ción y diseño de polític as públic as elaboradasen tomo a la preservación de dicho ecosistema.

Por último, las autoras conc luyen que este colectivo de ganaderos ha demostrado que a partir de un escenario político esta ble y de a poyo institucional pertinente, han sido capaces de realizar un uso sustentable de los recursos natura les por medio de la valorización de sus formas tra diciona les de producir, protegiend o las condic iones existentes en el bioma considerado como un bien común. Sumado a ello, la ganadería familiar incorpora uaspectos soc ioc ultura les y de la tra dición regional que conforman el proceso de comunaliza ción de la gestión del sistema socioecológico en el que están insertos, en la medida en que las 
acciones del colectivo buscan atendera ese colectivo de la forma másigualita ria posible». En el siguiente tra bajo Conflictos sociotemitoriales: la pesca artesanal como bien común en Península Valdés, Argentina, de las académicas e investiga doras Pía Valeria Rius y Lorena Álvarez Manníquez, se analiza el uso compartido de zonas de pesca artesanal en un área natural declarada «Patrimonio de la Humanidad». Por lo mismo, esta es una zona que está definida por el uso sustentable del recurso mańtimo, así como la relación entre el área oceánica y el espacio continental, ambos considerados como zonas intangibles, de uso sostenido restringido, de asenta mientos poblacionales y de zonas de rehabilitación natural y cultural.

La participación de investigadores junto a las comunidades de pescadores artesanales que proviene de larga data- permite la conforma ción de Mesas de Trabajo por zonasy en func ión de ello, se han obtenid o resulta dos signific a tivosen la actividad pesquera, considerando la sustentabilidad de los recursos en un teritorio deteminado. Ello a partir del establec imiento de nomas forma lese informales de buenas prácticas, las cuales se mantienen hasta los días actuales, a pesar de las presionesejercidas poragentes extemos y que imponen límites para la consolidación exitosa de esta experiencia.

Es dec ir, ta les prác tic as son inc umplidas por pesc a dores que no tienen permiso y que colocan en riesgo la sustentabilidad del recurso. Ello ha creado conflictos con las a utoridades provinciales, ya que se reconoce la debilidad de éstaspara rea liza rsusta reas de monitoreo, control y fisc a liza ción de lasá rea scomprendidasen el Plan de Manejo ela borado conjuntamente entre los diversos ac tores interesa dos. En consecuencia, la a plicación de la nomativa esconsiderada inefic az en la medida que solo afecta a lospescadores y mariscadores con permiso, dejando sin sanción a quienes usufructúan de este recurso a través de vías ajenas a las estipula das en los Planes de Manejo, coloc ando en riesgo los esfuerzos desplegados por la comunidad de pescadores para mantener el uso sustentable de este bien común.

Ello releva por lo tanto el debate sobre el uso de losbienescomunesy como bien apunta la autora, «sobre los principios de justicia y dispositivos de participación ciudadana» en un espac io temitorial en el cual se encuentran insertos los pesc a dores artesa na les a so cia dosen diversos grupos, los investiga dores y las autorida des provincia les. Por lo mismo, dic ho debate público representa una instancia superadora de las lógicas del mercado que emergen de la experiencia de interc a mbio y de reconocimiento mutuo existentes en el ámbito de la construc ción social presente en estascomunidades de pescadores.

Continuando este dossier, encontramos el aporte de Janett Vallejo Román y J uan Carlos Ro dríguez Torrent, titula do Bienes comunes, consenvación ambiental y economía campesina. Un estudio de caso en el Parque Nacional Cofre de Perote, Veracruz, México, en la cual se abordan las tensiones sobre el uso de bienescomunesnaturales-como lasáreas de alta biodiversidad- entre usuarios locales que han hecho un uso consuetudinario de los mismos bajo sistemas regula torios a utogestiona rios y las pretensiones de regula c ión esta tal superior 
que los asume como áreas de conservación de relevancia global.

Tomando como caso un parque nacional en México, se problematiza en tomo a los distintos usosy usuarios de la natura leza: por una parte, los proyectos de conservación sujeto a la nomativa de Área Nacional Protegida, y por la otra, poblaciones locales que hacen uso de la naturaleza como parte de su forma de vida. Se profundiza así en las controversias discursivas y prácticas en tomo a la gestión, diseño, planeación y gobemanza del bien común. El caso, convoca entonces a reflexionarsobre cómo generarcondiciones de reconocimiento e integración de las poblaciones locales que superen la oposición entre sociedad y biodiversidad -donde toda acción antrópica es siempre vista como negativa-. Esto implic a reconocer la historia adaptativa de las poblaciones humanas y su codependencia al cuidado y reproducción de los comunes.

En el séptimo y último artíc ulo de esta sección, la aca démica Ma rilia Veníssimo Veronese, de la UNISINOS, Brasil, nos entrega su contribuc ión titula da Economia solidária, saúde mental e arte/c ultura: promovendo a racionalidade política dos comuns, en la cual se propone realizar una valorización de los comunes a partir del derecho fundamental a acceder a los servicios públic os de salud mental y de su relación con la producción de arte y cultura, las que se difunden a través de prácticas económicas solidarias en redes de cooperación, reciprocidad y ayuda mutua. El estudio en cuestión fue rea liza do en dos comunida des ubicadas en dosciudades de Brasil, Rio de Janeiro y Porto Alegre.

La investiga c ión a borda las estra tegias de reha bilitac ión en salud mental de los usuarios de la Red de Atención Psic osocial (RAPS) por medio de a ctivida des grupales vinculantes y de la produc ción artística cultura I. Dicha producción se vincula a inicia tiva sque promueven el enraizamiento comunitario y la construcción de sentido entre los integrantes de las dos orga niza ciones estudia das.

El asociativismo generado en este proceso supone una práctica que supera la lógica mercantil y las leyes de valor imperantes en la sociedad, posibilitando o creando el espacio de a utonomía entre los dolientes psíquicos, modific ando su inserción en el mundo. De manera que «a autoestima y consciencia de sí pasan también por un proceso de modificación, algo que no sería posible fuera de loscircuitos recíproc os activa dos por el mismo». La a utora concluye que reciprocidad, cooperación y produc ción de nomas comunes están en la base del fenómeno a nalizado, a partir de la multiplicidad de participaciones en la promoción de ese común.

El presente número de la revista Polis se completa con otros cinco artículos de la sección Avancesy resulta dos de Investiga c ión y dosreseñas de produc cionesc ientífic aspublic a das recientemente. 